\title{
Introduction of Recirculatory Analysis into Portal and Systemic Concentration Difference Method
}

\author{
Shinya Ueda, Kiyoshi Yamaoka,* Yoneichi Samai, and Terumichi NaKagawa \\ Graduate School of Pharmaceutical Sciences, Kyoto University, Yoshida Shimoadachi-cho, Sakyo-ku, Kyoto 606-8501, \\ Japan. Received May 17, 2001; accepted July 17, 2001
}

\begin{abstract}
Recirculatory analysis was introduced into the portal and systemic concentration difference method with double dosing (PS-DD method), which is an evaluation system for the local intestinal and hepatic first-pass effect. 5-Fluorouracil (5-FU) and cephalexin (CEX) were selected as model drugs. A new recirculatory system was constructed to predict the time courses of a drug concentration in the systemic and portal bloods. Bioavailability $(F)$, local absorption ratio $\left(F_{\mathrm{a}}\right)$, hepatic recovery ratio $\left(F_{\mathrm{H}}\right)$, and local mean absorption time $\left(\bar{t}_{\mathrm{a}}\right)$ estimated by recirculatory analysis were close to those calculated by moment analysis with numerical integration. Using recirculatory analysis, the sampling period was considerably shortened and the sampling number was also reduced, which demonstrates that recirculatory analysis is useful in PS-DD method.
\end{abstract} kinetic

Key words recirculatory analysis; first-pass effect; intestinal absorption; hepatic metabolism; local moment analysis; pharmaco-

An in vivo experimental method was developed to determine the extent and rate of intestinal absorption of a drug into the portal system using the concentration difference between portal and systemic bloods (PS method). ${ }^{1-5)}$ As an extension of PS method, the intestinal and hepatic first-pass effects of a drug were evaluated by means of an intra-arterial dose followed by an oral dose to a single conscious rat in a short time interval (PS-DD method). ${ }^{6-8)}$ PS-DD method provides both the absolute bioavailability and the hepatic recovery ratio of a drug in a single animal, in addition to the local absorption ratio and time required to pass from the intestinal tract into the portal system. Local moment analysis in PS-DD method, which is model-independent, has been applied to evaluate intestinal and hepatic first-pass effects. Moment analysis, however, needs a long monitoring period, much sampling data and a large volume of blood to determine whole first-pass characteristics. Additionally, based on moment analysis, it is difficult to predict the time courses of a drug in systemic and portal bloods. The compartment models, which have widely been accepted, do not involve the flow rates of portal and total bloods which are considered to be important when discussing first-pass effects.

The concept of recirculatory pharmacokinetic analysis is that the observed blood concentration-time profile of a drug is expressed as a function of the infinite circulation of the local time profile generated by single passage through each organ in the body. ${ }^{9-11)}$ In the recirculatory theory, local drug disposition in organs is treated as unknown functions that are characterized by only their input-output behavior under the assumption of linear pharmacokinetic conditions. The recirculatory system based on the relationship between blood concentration-time profiles and local time profiles is usually expressed by Laplace-transformed equations. Therefore, it should be noted that there is difficulty in analytical inversion of the transformed equations into the corresponding real equations. In order to manipulate transformed equations, we developed a program for nonlinear least squares with fast inverse Laplace transform, MULTI(FILT) ${ }^{12)}$ The difference in the arterial and venous blood concentration-time profiles of metoprolol was analyzed by MULTI(FILT). ${ }^{13)}$ Furthermore, recirculatory theory was applied to analyses of not only global disposition but also local disposition such as enterohepatic circulation. ${ }^{14)}$ Thus, the concept "recirculatory" has been demonstrated to be useful to understand global pharmacokinetics in relation to local disposition of a drug.

In the present study, we attempted to apply recirculatory theory to evaluation of intestinal absorption and hepatic firstpass metabolism. 5-Fluorouracil (5-FU) and cephalexin (CEX) are selected as model drugs. 5-FU displays large inter-individual variation in absorption and disposition between rats ${ }^{15)}$ and between humans, ${ }^{16)}$ whereas CEX is perfectly absorbed from the intestinal tract into the portal system and is moved into the circulation system without firstpass metabolism through the liver. ${ }^{17)}$ Furthermore, we attempted to minimize the number of sampling points and to shorten the monitoring period by constructing a new pharmacokinetic model for PS-DD method, and the validity of the proposed model was confirmed using experimental data. ${ }^{6,7)}$

\section{THEORY}

Recirculatory Analysis Since a drug is administered into the femoral artery and systemic (venous or arterial) blood is collected from the same femoral artery, the theoretical equations for the Laplace transform of the systemic blood concentrations $\left(\widetilde{C}_{\text {i.a. }}^{\text {sys }}(s)\right)$ after intra-arterial bolus administration of the drug are given by ${ }^{11)}$ :

$$
\tilde{C}_{\text {i.a. }}^{\text {sys }}=\frac{D_{\text {i.a. }}}{Q}\left\{\tilde{f}_{\text {cyc }}(s)+\left(\tilde{f}_{\text {cyc }}(s)\right)^{2}+\left(\tilde{f}_{\text {cyc }}(s)\right)^{3}+\cdots\right\}=\frac{D_{\text {i.a. }}}{Q} \cdot \frac{\tilde{c}_{\text {cyc }}(s)}{f_{\text {cyc }}(s)}
$$

where $D_{\text {i.a }}$ is intra-arterial dose, $Q$ is total blood flow rate, and $\tilde{f}_{\text {cyc }}(s)$ is transfer function for systemic circulation.

The theoretical equations for the Laplace transform of systemic and portal blood concentrations after oral administration of a drug, $\widetilde{C}_{p . o .}^{\text {sys }}(s)$ and $\widetilde{C}_{p . o}^{\text {por }}(s)$ are given by following equations;

$$
\begin{aligned}
\tilde{C}_{p . o .}^{\text {sys }}(s)= & \frac{D_{p . o o}}{Q_{\mathrm{p}}} \tilde{f}_{\text {in }}(s) \cdot \tilde{f}_{\text {hep }}(s) \cdot \frac{Q_{\mathrm{p}}}{Q} \tilde{f}_{\text {cyc }}(s) \\
& +\frac{D_{p . o .}}{Q_{\mathrm{p}}} \tilde{f}_{\text {in }}(s) \cdot \tilde{f}_{\text {hep }}(s) \cdot \frac{Q_{\mathrm{p}}}{Q}\left\{\tilde{f}_{\text {cyc }}(s)\right\}^{2}+\cdots
\end{aligned}
$$




$$
\begin{aligned}
= & \frac{D_{p . o .}}{Q} \tilde{f}_{\text {in }}(s) \cdot \tilde{f}_{\text {hep }}(s) \cdot \frac{\tilde{f}_{\text {cyc }}(s)}{1-\tilde{f}_{\text {cyc }}(s)} \\
\tilde{C}_{p . o .}^{\text {por }}(s)= & \frac{D_{p . o .}}{Q_{\mathrm{p}}} \tilde{f}_{\text {in }}(s)+\frac{D_{p . o .}}{Q_{\mathrm{p}}} \tilde{f}_{\text {in }}(s) \cdot \tilde{f}_{\text {hep }}(s) \cdot \frac{Q_{\mathrm{p}}}{Q} \tilde{f}_{\text {cyc }}(s) \\
& +\frac{D_{p . o .}}{Q_{\mathrm{p}}} \tilde{f}_{\text {in }}(s) \cdot \tilde{f}_{\text {hep }}(s) \cdot \frac{Q_{\mathrm{p}}}{Q}\left\{\tilde{f}_{\text {cyc }}(s)\right\}^{2}+\cdots \\
= & \frac{D_{p . o .}}{Q_{\mathrm{p}}} \tilde{f}_{\text {in }}(s)+\frac{D_{p . o .}}{Q} \tilde{f}_{\text {in }}(s) \cdot \tilde{f}_{\text {hep }}(s) \cdot \frac{\tilde{f}_{\text {cyc }}(s)}{1-\tilde{f}_{\text {cyc }}(s)}
\end{aligned}
$$

Comparing Eqs. (2) and (3),

$$
\tilde{C}_{p . o .}^{\text {por }}(s)=\tilde{C}_{p . o .}^{\text {sys }}(s)+\frac{D_{p . o .}}{Q_{\mathrm{p}}} \tilde{f}_{\text {in }}(s)
$$

where $\tilde{f_{\text {in }}}(s)$ and $\tilde{f_{\text {hep }}}(s)$ are transfer functions expressing processes through the gastrointestinal tract to the portal system and through the liver, respectively. $\tilde{f}_{\text {cyc }}(s)$ is a weight function expressing processes through the blood circulation system inside the body. $D_{\text {p.o. }}$ is the administered dose through the oral route. $Q_{\mathrm{p}}$ and $Q$ are the portal blood flow rate and total blood flow rate, respectively.

\section{MATERIALS AND METHODS}

Chemicals 5-FU and 5-Bromouracil (5-BU as an internal standard) were purchased from Sigma Chemical Company (St. Louis, MO, U.S.A.). Cephalexin was purchased from Wako Pure Chemical Industries (Osaka Japan). Heparin was obtained from Novo Industries (Denmark). All other chemicals and reagents used were of analytical or HPLC grade.

Animal Experiments Healthy male Wistar rats, weighing 210-260 g, were purchased from Shimizu Experimental Materials Company, Ltd. (Kyoto, Japan) and maintained on standard chow and water ad libitum. All animals were starved for $16 \mathrm{~h}$, with free access of water, prior to the experiments. Under light ether anesthesia, the abdomen was opened by midline incision. The duodenum, drawn out from the incision, was fixed to expose the portal vein. Intramedic polyethylene tubing (PE10, Becton Dickinson, Sparks, MD, U.S.A.), as a cannula for portal vein, was inserted into the junction of the portal vein and the inferior pancreatic duodenal vein, and the tip of the catheter was placed close to the liver. The catheter was secured to the mesentery with a drop of cyanoacrylate adhesive (Aron Alpha; Sankyo, Tokyo, Japan). The free end of the catheter was exteriorized through a small puncture using an 18-gauge needle in the side abdominal wall. The right femoral artery of each rat was also cannulated, and the free side of the catheter was subcutaneously conducted and exteriorized at the back of the leg. These two cannulas were filled with heparinized saline solution $(100 \mathrm{U} / \mathrm{ml})$ and connected to a $1 \mathrm{ml}$ syringe (PLASTIPAK; Becton Dickinson, Sparks, MD, U.S.A.). Each rat was held in an animal cage (Bollman cage; Natsume, Tokyo Japan) and was allowed to recover from the ether anesthesia for more than $3 \mathrm{~h}$.

5 -FU dissolved in saline solution $(10 \mathrm{mg} / \mathrm{ml})$ was administrated orally $90 \mathrm{~min}$ after intra-arterial administration (10 $\mathrm{mg} / \mathrm{ml})$ at the same dosage $(30 \mathrm{mg} / \mathrm{kg})$. Blood specimens
(50 $\mu \mathrm{l})$ from both the femoral artery and portal vein were sampled at 5, 15, 30, 60, $90 \mathrm{~min}$ after an intra-arterial dose, and blood specimens $(50 \mu \mathrm{l})$ from both the femoral artery and the portal vein were taken at 5, 10, 15, 30, 45, 60, 90, 180 min after oral administration.

CEX dissolved in saline solution $(25 \mathrm{mg} / \mathrm{ml})$ was administrated orally $180 \mathrm{~min}$ after intra-arterial administration (25 $\mathrm{mg} / \mathrm{ml})$ at the same dosage $(50 \mathrm{mg} / \mathrm{kg})$. Blood specimens $(60 \mu \mathrm{l})$ from both the femoral artery and hepatic vein were sampled at 5, 15,30,60,120,180 min after an intra-arterial dose, and blood specimens $(60 \mu \mathrm{l})$ from both the femoral artery and the portal vein were taken at $15,30,45,60,90$, $120,180,300 \mathrm{~min}$ after oral administration. After centrifugation for $5 \mathrm{~min}$ at $2000 \boldsymbol{g}$, separated plasma samples were analyzed.

Assay Procedure The concentration of 5-FU in blood was determined by HPLC. The blood sample $(50 \mu \mathrm{l})$ was added to $250 \mu \mathrm{l}$ of internal standard solution $(1 \mu \mathrm{g} / \mathrm{ml}$ of 5 bromouracil) dissolved in $0.1 \mathrm{~m}$ phosphate buffer at $\mathrm{pH} 2.5$. The mixture was extracted three times with $750 \mu 1$ of ethyl acetate. The combined organic layers were evaporated under a nitrogen stream at $50^{\circ} \mathrm{C}$ and the residue was reconstituted with $250 \mu \mathrm{l}$ of mobile phase. A $100 \mu \mathrm{l}$ portion was injected into an HPLC system ( 800 series, Japan Spectroscopic, Tokyo, Japan) and a Chemcosorb 5-ODS-H reverse-phase column $(5 \mu \mathrm{m}, 150 \times 4.6 \mathrm{~mm}$ i.d., Chemco Scientific, Osaka, Japan). The detector wavelength, flow rate, and column temperature were set at $260 \mathrm{~nm}, 1.0 \mathrm{ml} / \mathrm{min}$, and $40^{\circ} \mathrm{C}$, respectively. The mobile phase consisted of $10 \mathrm{~mm}$ sodium acetate buffer ( $\mathrm{pH} 4.0)$ : methanol $(100: 1, \mathrm{v} / \mathrm{v})$. The chromatographic peaks were integrated with an electric integrator (Chromatopac C-R6A, Shimadzu, Kyoto, Japan). Using the peak area ratio to an internal standard ranging from 0.1 to 62.5 $\mu \mathrm{g} / \mathrm{ml}$ of 5-FU, calibration parameters were calculated. All correlation coefficients were greater than 0.999 .

In the preliminary experiment, 5-FU concentrations were compared between portal and systemic bloods from 0 to 90 min after intra-arterial administration. It was found that the concentration difference between portal and arterial bloods was negligibly small.

The concentration of CEX in plasma was determined by HPLC. To determine the concentration of CEX in plasma, $30 \mu \mathrm{l}$ of plasma was vortex-mixed vigorously for $30 \mathrm{~s}$ with $90 \mu \mathrm{l}$ of methanol then centrifuged at $2000 \mathrm{~g}$ for $5 \mathrm{~min}$. A $15 \mu \mathrm{l}$ portion was injected into an HPLC system (LC-10A, Shimadzu, Kyoto, Japan) and a Capcell Pak $\mathrm{C}_{18}$ column ( $5 \mu \mathrm{m}, 250 \times 4.6 \mathrm{~mm}$ i.d., Shiseido, Tokyo, Japan). The detector wavelength, flow rate, and column temperature were set at $264 \mathrm{~nm}, 1.0 \mathrm{ml} / \mathrm{min}$, and $40^{\circ} \mathrm{C}$, respectively. The mobile phase was $\mathrm{H}_{2} \mathrm{O}-\mathrm{MeOH}(7: 2 \mathrm{v} / \mathrm{v}$, adjusted to $\mathrm{pH} 3.05$ with acetic acid). The chromatographic peaks were integrated with an electric integrator (Chromatopac C-R6A, Shimadzu, Kyoto, Japan). Using the peak area from 0.5 to $100 \mu \mathrm{g} / \mathrm{ml}$ of CEX, calibration parameters were calculated. All correlation coefficients were greater than 0.999 .

In the preliminary experiment, CEX concentrations were compared between portal and systemic bloods from 0 to 180 min after intra-arterial administration. It was found that the concentration difference between portal and arterial bloods was negligibly small. 


\section{NUMERICAL CALCULATIONS}

$\tilde{f}_{\text {cyc }}(s)$ is assumed to be mono-exponential.

$$
\tilde{f}_{\mathrm{cyc}}(s)=\frac{F_{\mathrm{c}} k_{\mathrm{c}}}{s+k_{\mathrm{c}}}
$$

where $k_{\mathrm{c}}$ is the elimination rate constant from systemic circulation, and $F_{\mathrm{c}}$ is the elimination ratio from systemic circulation. Substituting Eq. (5) into Eq. (1),

$$
\tilde{C}_{\text {i.a. }}^{\text {sys }}=\frac{D_{\text {i.a. }}}{Q} \cdot \frac{\tilde{f}_{\text {cyc }}(s)}{1-\tilde{f}_{\text {cyc }}(s)}=\frac{D_{\text {i.a. }}}{Q} \cdot \frac{\frac{F_{\mathrm{c}} k_{\mathrm{c}}}{s+k_{\mathrm{c}}}}{1-\frac{F_{\mathrm{c}} k_{\mathrm{c}}}{s+k_{\mathrm{c}}}}=\frac{D_{\mathrm{i} \text {.a. }}}{Q} \cdot \frac{F_{\mathrm{c}} k_{\mathrm{c}}}{s+\left(1-F_{\mathrm{c}}\right) k_{\mathrm{c}}}
$$

The mean hepatic transit time is predicted to be negligible, and $\tilde{f}_{\text {hep }}(s)$ is assumed as

$$
\tilde{f}_{\text {hep }}(s)=F_{\mathrm{H}} \tilde{\delta}(t)
$$

$\tilde{f}_{\text {in }}(s)$ is approximated by the following equation.

$$
\tilde{f}_{\text {in }}(s)=\frac{4 k_{\mathrm{a}}^{2} F_{\mathrm{a}}}{\left(s+2 k_{\mathrm{a}}\right)^{2}} \cdot e^{-s T}
$$

The derivation of Eq. (8) is given later. Substituting Eqs. (5), (7) and (8) into Eqs. (2) and (3),

$$
\begin{aligned}
\tilde{C}_{p . o .}^{\text {sys }}(s)= & \frac{D_{p . o .}}{Q} \tilde{f}_{\mathrm{in}}(s) \cdot \tilde{f}_{\mathrm{hep}}(s) \cdot \frac{\tilde{f}_{\mathrm{cyc}}(s)}{1-\tilde{f}_{\mathrm{cyc}}(s)} \\
= & \frac{D_{p . o .}}{Q} \cdot \frac{4 k_{\mathrm{a}}^{2} F_{\mathrm{a}}}{\left(s+2 k_{\mathrm{a}}\right)^{2}} \cdot F_{\mathrm{h}} \cdot \frac{\frac{F_{\mathrm{c}} k_{\mathrm{c}}}{s+k_{\mathrm{c}}}}{1-\frac{F_{\mathrm{c}} k_{\mathrm{c}}}{s+k_{\mathrm{c}}}} e^{-s T} \\
= & \frac{D_{p . o .}}{Q} \cdot \frac{4 k_{\mathrm{a}}^{2} F_{\mathrm{a}}}{\left(s+2 k_{\mathrm{a}}\right)^{2}} \cdot F_{\mathrm{h}} \cdot \frac{F_{\mathrm{c}} k_{\mathrm{c}}}{s+\left(1-F_{\mathrm{c}}\right) k_{\mathrm{c}}} \cdot e^{-s T} \\
\tilde{C}_{p . o .}^{\text {por }}(s)= & \frac{D_{p . o .}}{Q} \cdot \frac{4 k_{\mathrm{a}}^{2} F_{\mathrm{a}}}{\left(s+2 k_{\mathrm{a}}\right)^{2}} \cdot F_{\mathrm{h}} \cdot \frac{F_{\mathrm{c}} k_{\mathrm{c}}}{s+\left(1-F_{\mathrm{c}}\right) k_{\mathrm{c}}} \cdot e^{-s T} \\
& +\frac{D_{p . o .}}{Q} \cdot \frac{4 k_{\mathrm{a}}^{2} F_{\mathrm{a}}}{\left(s+2 k_{\mathrm{a}}\right)^{2}} \cdot e^{-s T} \\
=\left(\frac{D_{p . o .}}{Q}\right. & \left.\frac{4 k_{\mathrm{a}}^{2} F_{\mathrm{a}}}{\left(s+2 k_{\mathrm{a}}\right)^{2}} \cdot F_{\mathrm{h}} \cdot \frac{F_{\mathrm{c}} k_{\mathrm{c}}}{s+\left(1-F_{\mathrm{c}}\right) k_{\mathrm{c}}}+\frac{D_{p . o .}}{Q_{\mathrm{p}}} \cdot \frac{4 k_{\mathrm{a}}^{2} F_{\mathrm{a}}}{\left(s+2 k_{\mathrm{a}}\right)^{2}}\right) \cdot e^{-s T}
\end{aligned}
$$

The drug concentration in portal vein after oral administration, $C_{p . o .}^{\text {por }}(t)$ given by Eq. (4) is rewritten as

$$
C_{p . o .}^{\mathrm{por}}(t)=C_{p . o .}^{\mathrm{sys}}(t)+\Delta C(t)
$$

where $C_{p . o .}^{\text {sys }}(t)$ is drug concentration in systemic vein and $\Delta C(t)$ is loaded concentration absorbed from the intestinal tract to the portal system, respectively.

If a drug is assumed to be absorbed according to firstorder absorption rate, the loaded concentration is given by

$$
\Delta C(t)=\Delta C_{0} \frac{k_{1}}{k_{1}-k_{2}}\left(e^{-k_{2}(t-T)}-e^{-k_{1}(t-T)}\right)
$$

where $\Delta C_{0}$ is initial drug concentration difference between portal vein and systemic vein at oral administration $(t=0), k_{1}$ and $k_{2}$ are transition rates from intestinal tract to portal system, and $T$ is the lag time from stomach to intestine, respectively. Since $k_{1}$ and $k_{2}$ were almost equal, Eq. (12) is reduced to Eq. (13) according to De L'Hopital principal.

$$
\Delta C(t)=\Delta C_{0} k_{1}(t-T) e^{-k_{1}(t-T)}
$$

The absorption amount from intestinal tract into the portal system is calculated by the following equation based on Fick's principal. ${ }^{1,3-8)}$

$$
F_{\mathrm{a}} \cdot D_{p . o .}=Q_{\mathrm{p}} \cdot \int_{0}^{\infty}\left(C_{p . o .}^{\mathrm{por}}(t)-C_{p . o .}^{\mathrm{sys}}(t)\right) d t
$$

where $F_{\mathrm{a}}$ is absorption ratio from intestinal tract into the portal system and $D_{\text {p.o. }}$ is orally administered drug amount and $Q_{\mathrm{p}}$ is blood flow rate of portal vein, respectively. From Eqs. (11), (13) and (14), $\Delta C_{0}$ is given by

$$
\Delta C_{0}=\frac{F_{\mathrm{a}} D_{p . o .} k_{1}}{Q_{\mathrm{p}}}
$$

The mean absorption time (MAT) of a drug is calculated by the following equation

$$
M A T=\frac{1}{k_{\mathrm{a}}}=\bar{t}_{\mathrm{a}}+\bar{t}_{\mathrm{H}}
$$

where $k_{\mathrm{a}}$ is absorption rate from intestinal tract to systemic circulation and $\bar{t}_{\mathrm{a}}$ is mean local absorption time from intestinal tract into the portal system and $\bar{t}_{\mathrm{H}}$ is mean hepatic transit time. Since $\bar{t}_{\mathrm{H}}$ is considered to be negligible, $M A T$ is equal to $\bar{t}_{\mathrm{a}} \cdot{ }^{3,4,6,8)} \bar{t}_{\mathrm{a}}$ is calculated by the following equation

$$
\bar{t}_{\mathrm{a}}=\frac{1}{k_{1}}+\frac{1}{k_{2}} \approx \frac{2}{k_{1}}
$$

From Eqs. (11), (16), and (17), the drug concentration in portal vein after oral administration $\left(C_{\text {p.o. }}^{\text {por }}(t)\right)$ is given by

$$
C_{p . o .}^{\text {por }}(t)=C_{p . o .}^{\text {sys }}(t)+\frac{4 k_{\mathrm{a}}^{2} F_{\mathrm{a}} D_{p . o .}}{Q_{\mathrm{p}}}(t-T) e^{-2 k_{\mathrm{a}}(t-T)}
$$

Laplace-transforming Eq. (18) gives Eq.(10).

Eqs. (6), (9) and (10) were fitted to the blood concentration profiles of 5-FU and the plasma concentration of CEX by means of MULTI(FILT). The values of $Q(=40.0 \mathrm{ml} / \mathrm{min})$ and $Q_{\mathrm{p}}(15.3 \mathrm{ml} / \mathrm{min})$ per $250 \mathrm{~g}$ rat were adopted from the literatures. ${ }^{6-8,18,19)} F$ was calculated by $F_{\mathrm{a}} \cdot F_{\mathrm{H}} \cdot F, F_{\mathrm{a}}, F_{\mathrm{H}}$, and $\bar{t}_{\mathrm{a}}$ estimated by recirculatory analysis were compared to those calculated by moment analysis with numerical integration in the literatures. $^{6,7)}$

The effect of reducing the sampling data after oral administration to the local absorption parameters, $F, F_{\mathrm{a}}, F_{\mathrm{H}}, \bar{t}_{\mathrm{a}}, k_{\mathrm{c}}$ and $F_{\mathrm{c}}$ were simulated on MS-DOS computers. In both cases of 5-FU and CEX, data points for the rear part of the time course were neglected in succession, firstly 1 point, 2 points, 3 points and finally 4 points, and $F, F_{\mathrm{a}}, F_{\mathrm{H}}, \bar{t}_{\mathrm{a}}, k_{\mathrm{c}}$ and $F_{\mathrm{c}}$ were calculated for each time course.

\section{RESULTS}

Figure 1 presents representative time courses for 5-FU and CEX for one rat and the predicted curves based on Eqs. (6), (9) and (10), parameters of which were estimated by curve fitting with MULTI(FILT).

Table 1 shows $F, F_{\mathrm{a}}, F_{\mathrm{H}}, \bar{t}_{\mathrm{a}}, k_{\mathrm{c}}$ and $F_{\mathrm{c}}$ for 5-FU $(n=5)$ based on recirculatory model analysis and $F, F_{\mathrm{a}}, F_{\mathrm{H}}$, and $\bar{t}_{\mathrm{a}}$ for 5-FU $(n=5)$ based on moment integration analysis. By 
$5-\mathrm{FU}$

0
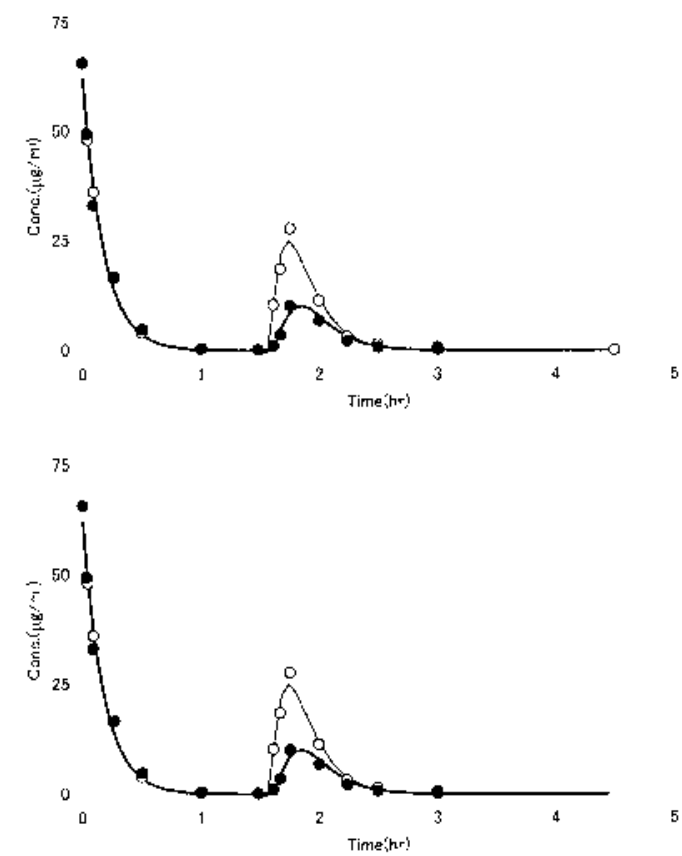

2

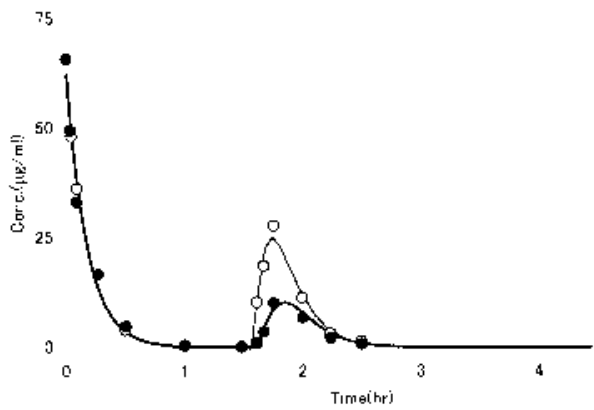

3
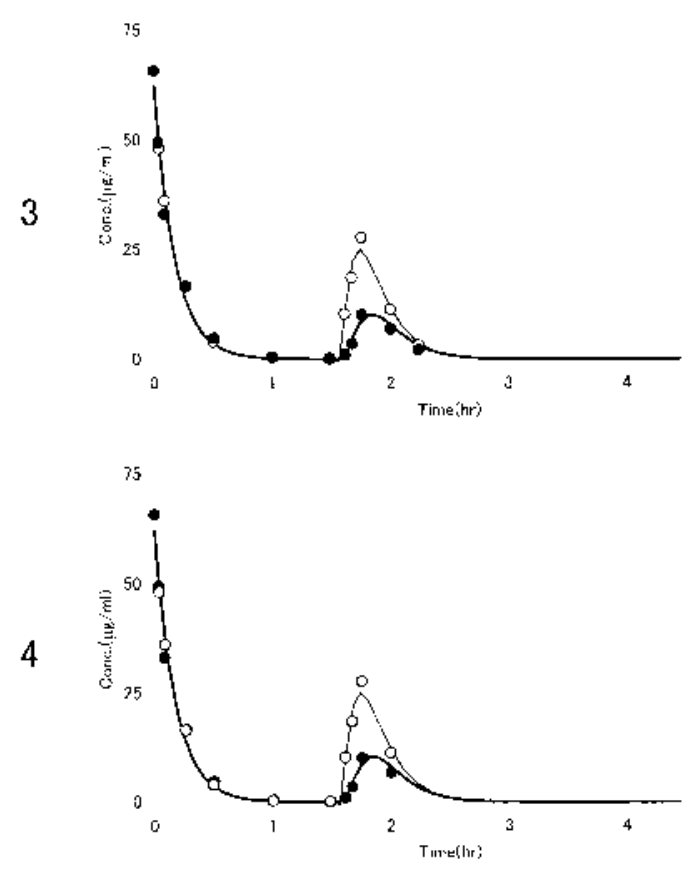

CEX

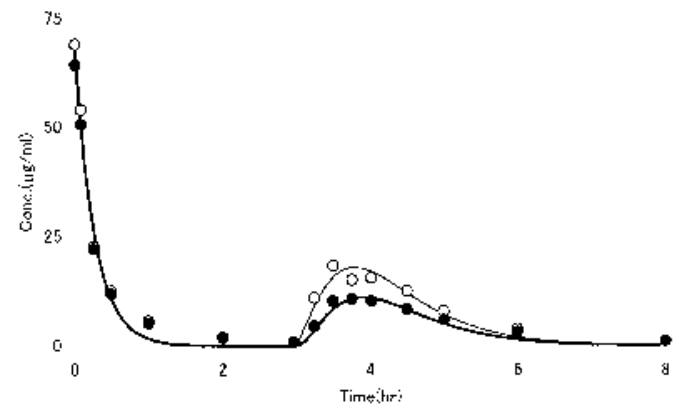

75
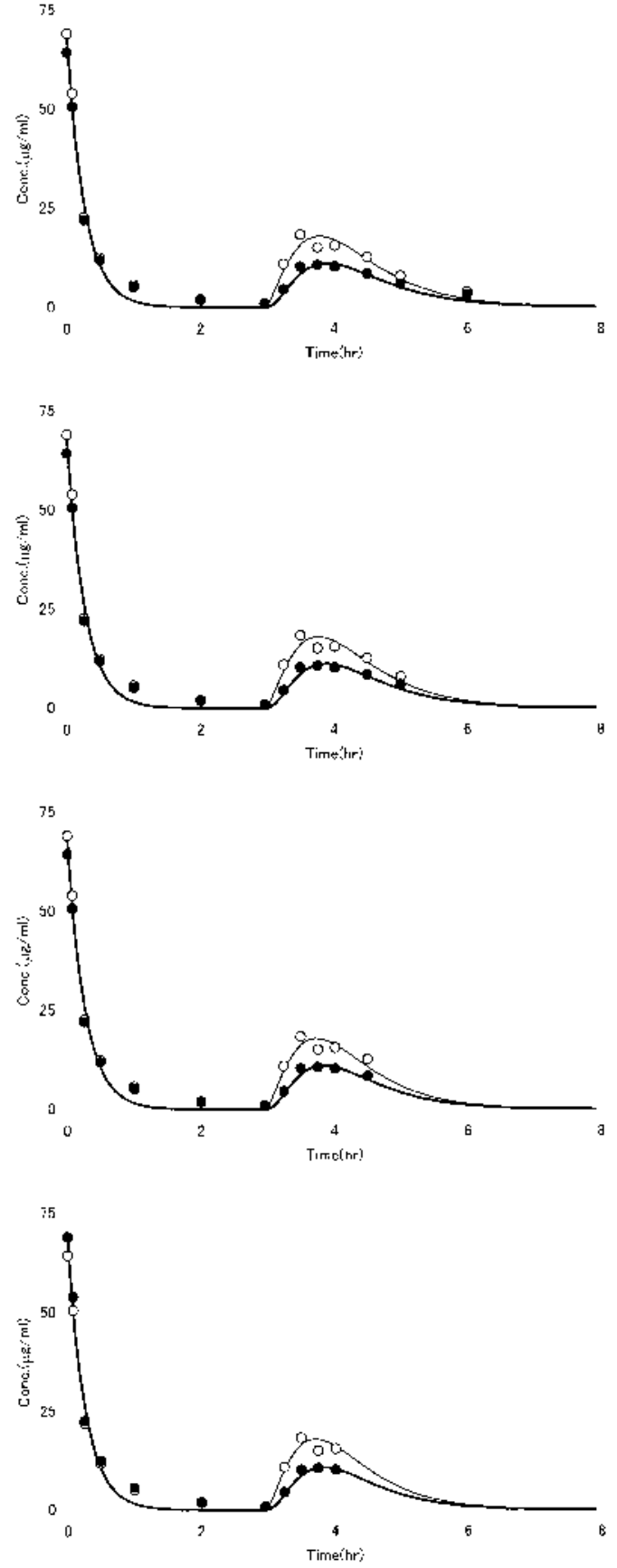

Fig. 1. Representative Time Courses for 5-FU and CEX in Rat, and the Predicted Curves

0, 1, 2, 3 and 4 of 5-FU and CEX mean the time courses using full points, neglecting the last 1-point, 2-points, 3-points and 4points, respectively. The open circles and closed circles for 5-FU and CEX are the concentrations of portal blood and arterial blood, respectively. 
Table 1. Comparison of Local Absorption Parameters of 5-FU after Intra-arterial Administration and Oral Administration; Recirculatory Analysis vs. Moment Analysis

\begin{tabular}{|c|c|c|c|c|c|c|}
\hline \multirow{2}{*}{ Recirculatory analysis } & \multicolumn{5}{|c|}{ Rat } & \multirow{2}{*}{ Mean \pm S.D. } \\
\hline & 1 & 2 & 3 & 4 & 5 & \\
\hline$F$ & 0.414 & 0.228 & 0.451 & 0.620 & 0.146 & $0.372 \pm 0.188$ \\
\hline$F_{\mathrm{a}}$ & 0.693 & 0.421 & 0.664 & 0.765 & 0.639 & $0.637 \pm 0.130$ \\
\hline$F_{\mathrm{H}}$ & 0.598 & 0.543 & 0.679 & 0.810 & 0.228 & $0.572 \pm 0.217$ \\
\hline $\bar{t}_{\mathrm{a}}(\mathrm{h})$ & 0.437 & 0.428 & 0.219 & 0.469 & 0.753 & $0.461 \pm 0.191$ \\
\hline$k_{\mathrm{c}}(1 / \mathrm{h})$ & 24.8 & 22.2 & 26.4 & 31.1 & 27.4 & $26.4 \pm 3.3$ \\
\hline$F_{\mathrm{c}}$ & 0.745 & 0.761 & 0.786 & 0.754 & 0.752 & $0.760 \pm 0.016$ \\
\hline \multirow{2}{*}{ Moment analysis } & \multicolumn{5}{|c|}{ Rat } & \multirow{2}{*}{ Mean士S.D. } \\
\hline & 1 & 2 & 3 & 4 & 5 & \\
\hline$F$ & 0.309 & 0.181 & 0.356 & 0.427 & 0.139 & $0.282 \pm 0.120$ \\
\hline$F_{\mathrm{a}}$ & 0.715 & 0.460 & 0.823 & 0.869 & 0.607 & $0.695 \pm 0.166$ \\
\hline$F_{\mathrm{H}}$ & 0.432 & 0.393 & 0.433 & 0.491 & 0.229 & $0.396 \pm 0.099$ \\
\hline $\bar{t}_{\mathrm{a}}(\mathrm{h})$ & 0.507 & 0.538 & 0.393 & 0.417 & 0.890 & $0.549 \pm 0.200$ \\
\hline
\end{tabular}

Table 2. Comparison of Local Absorption Parameters of CEX after Intra-arterial Administration and Oral Administration; Recirculatory Analysis vs. Moment Analysis

\begin{tabular}{|c|c|c|c|c|c|}
\hline \multirow{2}{*}{ Recirculatory analysis } & \multicolumn{4}{|c|}{ Rat } & \multirow{2}{*}{ Mean士S.D. } \\
\hline & 1 & 2 & 3 & 4 & \\
\hline$F$ & 1.24 & 1.25 & 0.94 & 1.06 & $1.12 \pm 0.15$ \\
\hline$F_{\mathrm{a}}$ & 0.976 & 1.17 & 0.962 & 1.08 & $1.05 \pm 0.10$ \\
\hline$F_{\mathrm{H}}$ & 1.28 & 1.06 & 0.977 & 0.986 & $1.08 \pm 0.14$ \\
\hline $\bar{t}_{\mathrm{a}}(\mathrm{h})$ & 1.28 & 1.09 & 1.22 & 1.14 & $1.18 \pm 0.08$ \\
\hline$k_{\mathrm{c}}(1 / \mathrm{h})$ & 21.9 & 24.0 & 22.8 & 24.0 & $23.2 \pm 1.0$ \\
\hline \multirow[t]{2}{*}{$F_{\mathrm{c}}$} & 0.831 & 0.834 & 0.844 & 0.845 & $0.839 \pm 0.007$ \\
\hline & \multicolumn{4}{|c|}{ Rat } & \multirow{2}{*}{ Mean士S.D. } \\
\hline Moment analysis & 1 & 2 & 3 & 4 & \\
\hline$F$ & 1.04 & 1.12 & 1.16 & 1.00 & $1.08 \pm 0.07$ \\
\hline$F_{\mathrm{a}}$ & 0.837 & 1.11 & 0.856 & 0.944 & $0.937 \pm 0.125$ \\
\hline$F_{\mathrm{H}}^{\mathrm{a}}$ & 1.24 & 1.01 & 1.36 & 1.06 & $1.17 \pm 0.16$ \\
\hline $\bar{t}_{\mathrm{a}}(\mathrm{h})$ & 1.63 & 1.34 & 2.03 & 1.19 & $1.55 \pm 0.37$ \\
\hline
\end{tabular}

using recirculatory analysis, $F, F_{\mathrm{a}}, F_{\mathrm{H}}, \bar{t}_{\mathrm{a}}, k_{\mathrm{c}}$, and $F_{\mathrm{c}}$ were $0.372 \pm 0.188,0.637 \pm 0.130,0.572 \pm 0.217,0.461 \pm 0.191 \mathrm{~h}$, $26.4 \pm 3.3 \mathrm{~h}^{-1}$, and $0.760 \pm 0.016$, respectively. By using moment analysis, $F, F_{\mathrm{a}}, F_{\mathrm{H}}$, and $\bar{t}_{\mathrm{a}}$ were $0.282 \pm 0.120,0.695 \pm$ $0.166,0.396 \pm 0.099$, and $0.549 \pm 0.200 \mathrm{~h}$, respectively. The differences between parameters from recirculatory analysis and moment analysis were insignificant by one way analysis of variance (ANOVA) (5\%). Table 2 shows $F, F_{\mathrm{a}}, F_{\mathrm{H}}, \bar{t}_{\mathrm{a}}, k_{\mathrm{c}}$, and $F_{\mathrm{c}}$ for CEX $(n=4)$ based on the recirculatory analysis and $F, F_{\mathrm{a}}, F_{\mathrm{H}}$, and $\bar{t}_{\mathrm{a}}$ for $\mathrm{CEX}(n=4)$ based on the moment analysis. By using the recirculatory analysis, $F, F_{\mathrm{a}}, F_{\mathrm{H}}, \bar{t}_{\mathrm{a}}, k_{\mathrm{c}}$, and $F_{\mathrm{c}}$ were $1.12 \pm 0.15,1.05 \pm 0.10,1.08 \pm 0.14$, and $1.18 \pm$ $0.08 \mathrm{~h}, 23.2 \pm 1.0 \mathrm{~h}^{-1}$, and $0.839 \pm 0.007$, respectively. By using the moment analysis, $F, F_{\mathrm{a}}, F_{\mathrm{H}}$, and $\bar{t}_{\mathrm{a}}$, were $1.08 \pm 0.07$, $0.937 \pm 0.125,1.17 \pm 0.16,1.55 \pm 0.37 \mathrm{~h}$, respectively. The differences between parameters from recirculatory analysis and moment analysis were insignificant by ANOVA (5\%).

Table 3 shows the effect of a shortened monitoring period and minimized sampling points on the local absorption and disposition parameters of 5-FU based on recirculatory analysis and moment analysis. Based on recirculatory analysis, $F$, $F_{\mathrm{a}}, F_{\mathrm{H}}, \bar{t}_{\mathrm{a}}, k_{\mathrm{c}}$, and $F_{\mathrm{c}}$ of the time courses where the last 4 sampling points were at most neglected and the sampling period was at least $0.5 \mathrm{~h}$ were $0.490 \pm 0.287,0.816 \pm 0.322,0.584 \pm$ $0.178, \quad 0.585 \pm 0.271 \mathrm{~h}, 26.4 \pm 3.3 \mathrm{~h}^{-1}$, and $0.760 \pm 0.016$, respectively. The differences in parameters between the first column and the other columns were insignificant by $\operatorname{ANOVA}(5 \%)$. Based on moment analysis, $F, F_{\mathrm{a}}, F_{\mathrm{H}}$, and $\bar{t}_{\mathrm{a}}$, for the same time courses where the last 3 sampling points were at most neglected and the sampling period was at least $0.75 \mathrm{~h}$, were $0.366 \pm 0.198,0.618 \pm 0.118,0.601 \pm 0.355$, and $0.215 \pm 0.407 \mathrm{~h}$, respectively. When the last 3 points were neglected, moment parameters of one rat were not calculated because of divergence. When 4 points were neglected, moment parameters for all rats were not obtained.

Table 4 shows data for CEX. Based on recirculatory analysis, $F, F_{\mathrm{a}}, F_{\mathrm{H}}, \bar{t}_{\mathrm{a}}, k_{\mathrm{c}}$, and $F_{\mathrm{c}}$ for time courses where the last 4 sampling points were at most neglected, and the sam- 
Table 3. The Effect of Shortened Sampling Time and Minimized Sampling Point after Oral Administration to Local Absorption Parameters of 5-FU Recirculatory analysis

\begin{tabular}{|c|c|c|c|c|c|}
\hline Sampling time and points & $3 \mathrm{~h}, 8$ points & $1.5 \mathrm{~h}, 7$ points & $1 \mathrm{~h}, 6$ points & $0.75 \mathrm{~h}, 5$ points & $0.5 \mathrm{~h}, 4$ points \\
\hline$F$ & $0.372 \pm 0.188$ & $0.332 \pm 0.108$ & $0.346 \pm 0.152$ & $0.356 \pm 0.187$ & $0.490 \pm 0.287$ \\
\hline$F_{\mathrm{a}}$ & $0.637 \pm 0.130$ & $0.663 \pm 0.155$ & $0.668 \pm 0.144$ & $0.676 \pm 0.249$ & $0.816 \pm 0.322$ \\
\hline$F_{\mathrm{H}}^{a}$ & $0.572 \pm 0.217$ & $0.506 \pm 0.139$ & $0.519 \pm 0.186$ & $0.508 \pm 0.177$ & $0.584 \pm 0.178$ \\
\hline $\bar{t}_{\mathrm{a}}(\mathrm{h})$ & $0.461 \pm 0.191$ & $0.503 \pm 0.283$ & $0.459 \pm 0.243$ & $0.451 \pm 0.148$ & $0.585 \pm 0.271$ \\
\hline$k_{\mathrm{c}}(1 / \mathrm{h})$ & $26.4 \pm 3.3$ & $26.4 \pm 3.3$ & $26.4 \pm 3.3$ & $26.4 \pm 3.3$ & $26.4 \pm 3.3$ \\
\hline$F_{\mathrm{c}}$ & $0.760 \pm 0.016$ & $0.761 \pm 0.015$ & $0.760 \pm 0.016$ & $0.760 \pm 0.016$ & $0.760 \pm 0.016$ \\
\hline \multicolumn{6}{|l|}{ Moment analysis } \\
\hline Sampling time and points & $3 \mathrm{~h}, 8$ points & $1.5 \mathrm{~h}, 7$ points & $1 \mathrm{~h}, 6$ points & $0.75 \mathrm{~h}, 5$ points & $0.5 \mathrm{~h}, 4$ points \\
\hline$F$ & $0.282 \pm 0.120$ & $0.281 \pm 0.115$ & $0.290 \pm 0.116$ & $0.366 \pm 0.198 *$ & ${ }^{a)}$ \\
\hline$F_{\mathrm{a}}$ & $0.695 \pm 0.166$ & $0.687 \pm 0.172$ & $0.631 \pm 0.149$ & $0.618 \pm 0.118 *$ & ${ }_{-a)}$ \\
\hline$F_{\mathrm{H}}$ & $0.396 \pm 0.099$ & $0.402 \pm 0.103$ & $0.450 \pm 0.097$ & $0.601 \pm 0.355^{*}$ & $-^{a)}$ \\
\hline $\bar{t}_{\mathrm{a}}(\mathrm{h})$ & $0.549 \pm 0.200$ & $0.531 \pm 0.187$ & $0.427 \pm 0.177$ & $0.215 \pm 0.407 *$ & $-^{a)}$ \\
\hline
\end{tabular}

Each value represents mean \pm S.D. $(n=5)$ except $*(n=4)$. a) Not obtained.

Table 4. The Effect of Shortened Sampling Time and Minimized Sampling Point after Oral Administration to Local Absorption Parameters of CEX Recirculatory analysis

\begin{tabular}{|c|c|c|c|c|c|}
\hline Sampling time and points & $5 \mathrm{~h}, 8$ points & $3 \mathrm{~h}, 7$ points & $2 \mathrm{~h}, 6$ points & $1.25 \mathrm{~h}, 5$ points & $1 \mathrm{~h}, 4$ points \\
\hline$F$ & $1.12 \pm 0.15$ & $1.09 \pm 0.13$ & $1.13 \pm 0.15$ & $1.03 \pm 0.16$ & $0.940 \pm 0.099$ \\
\hline$F_{\mathrm{a}}$ & $1.05 \pm 0.10$ & $1.01 \pm 0.10$ & $1.05 \pm 0.10$ & $0.917 \pm 0.118$ & $0.904 \pm 0.139$ \\
\hline$F_{\mathrm{H}}$ & $1.08 \pm 0.14$ & $1.08 \pm 0.13$ & $1.08 \pm 0.18$ & $1.13 \pm 0.17$ & $1.05 \pm 0.15$ \\
\hline $\bar{t}_{\mathrm{a}}(\mathrm{h})$ & $1.18 \pm 0.08$ & $1.15 \pm 0.09$ & $1.20 \pm 0.11$ & $1.05 \pm 0.08$ & $0.966 \pm 0.064 *$ \\
\hline$k_{\mathrm{c}}(1 / \mathrm{h})$ & $23.2 \pm 1.0$ & $22.4 \pm 1.0$ & $22.4 \pm 1.0$ & $22.4 \pm 1.0$ & $22.7 \pm 0.7$ \\
\hline$F_{\mathrm{c}}$ & $0.839 \pm 0.007$ & $0.838 \pm 0.010$ & $0.838 \pm 0.009$ & $0.838 \pm 0.009$ & $0.836 \pm 0.005$ \\
\hline \multicolumn{6}{|l|}{ Moment analysis } \\
\hline Sampling time and points & $5 \mathrm{~h}, 8$ points & $3 \mathrm{~h}, 7$ points & $2 \mathrm{~h}, 6$ points & $1.25 \mathrm{~h}, 5$ points & $1 \mathrm{~h}, 4$ points \\
\hline$F$ & $1.08 \pm 0.07$ & $1.07 \pm 0.09$ & $1.10 \pm 0.11$ & $1.29 \pm 0.23$ & -a) $^{a}$ \\
\hline$F_{\mathrm{a}}$ & $0.937 \pm 0.125$ & $0.864 \pm 0.150$ & $1.01 \pm 0.44$ & $2.27 \pm 1.97$ & a) $^{a}$ \\
\hline$F_{\mathrm{H}}^{\mathrm{a}}$ & $1.17 \pm 0.16$ & $1.27 \pm 0.26$ & $1.29 \pm 0.62$ & $1.14 \pm 1.06$ & -a) $^{a}$ \\
\hline $\bar{t}_{\mathrm{a}}^{\mathrm{H}}(\mathrm{h})$ & $1.55 \pm 0.37$ & $1.16 \pm 0.04$ & $1.31 \pm 1.74$ & $3.11 \pm 4.05$ & $-^{a)}$ \\
\hline
\end{tabular}

Each value represents mean \pm S.D. $(n=4) *$ Significant at $5 \%$ between 8 points data and 4 points data. $a)$ Not obtained.

pling period was at least $1 \mathrm{~h}$, were $0.940 \pm 0.099,0.904 \pm$ $0.139,1.05 \pm 0.15,0.966 \pm 0.064 \mathrm{~h}, 22.7 \pm 0.7 \mathrm{~h}^{-1}$, and $0.836 \pm$ 0.005 , respectively. The differences in parameters between the first column and the other columns were insignificant except for $\bar{t}_{\mathrm{a}}$ in last column, by ANOVA(5\%). Based on moment analysis, $F, F_{\mathrm{a}}, F_{\mathrm{H}}$, and $\bar{t}_{\mathrm{a}}$ of the same time courses where the last 3 sampling points were at most neglected and the sampling period was at least $1.25 \mathrm{~h}$, were $1.29 \pm 0.23$, $2.27 \pm 1.97,1.14 \pm 1.06$, and $3.11 \pm 4.05 \mathrm{~h}$, respectively. When the last 4 points were neglected, moment parameters for all rats were not obtained.

\section{DISCUSSION}

In both cases of 5-FU and CEX, $F, F_{\mathrm{a}}, F_{\mathrm{H}}$, and $\bar{t}_{\mathrm{a}}$ for each rat based on recirculatory analysis were close to the corresponding parameters obtained from the same rat based on moment analysis (Tables 1,2). Moment analysis with numerical integration is a model-independent method, and can be applied to any drugs that show various local absorption and disposition kinetics. However, to gain proper moment values, moment analysis needs a long sampling period and much sampling data. Thus, moment analysis is applicable only to drugs with quite rapid absorption and elimination kinetics. Furthermore, it is difficult to predict the time courses of drug in systemic blood and portal blood. In the present report, we have developed a new analysis method based on recirculatory analysis to minimize the sampling points and to shorten the sampling period by constructing a proper model system. We aimed at reducing the sampling times and the monitoring period after oral administration using dispositional information of the time course following intra-arterial administration. In both cases of 5-FU and CEX, properly estimating $F, F_{\mathrm{a}}, F_{\mathrm{H}}$, and $\bar{t}_{\mathrm{a}}$ requires a sampling period to be the same as the mean resident time (MRT) after oral administration that is calculated using the time course after oral administration. Based on moment analysis, it was difficult to calculate accurately $F$, $F_{\mathrm{a}}, F_{\mathrm{H}}$, and $\bar{t}_{\mathrm{a}}$ according to the blood sampling schedule above.

In conclusion, local absorption and disposition parameters, 
$F, F_{\mathrm{a}}, F_{\mathrm{H}}$, and $\bar{t}_{\mathrm{a}}$, estimated by recirculatory analysis are close to those calculated by moment analysis, which demonstrates that the proposed recirculatory system is accurate. Recirculatory analysis offers the predicted time courses of drug in systemic and portal bloods. Using the recirculatory system, the sampling period can be shortened and the sampling points can be reduced.

\section{REFERENCES AND NOTES}

1) Tabata K., Yamaoka K., Fukuyama T., Nakagawa T., Pharm. Res., 12, $880-883$ (1995).

2) Hoffman D. J., Seifert T., Borre A., Nellans H. N., Pharm. Res., 12, 889-894 (1995)

3) Fujieda Y., Yamaoka K., Ito T., Nakagawa T., Pharm. Res., 13, 12011204 (1996)

4) Sawai Y., Yamaoka K., Takemura A., Nakagawa T., J. Pharm. Sci., 86, 1269-1272 (1997)

5) Sawai Y., Yamaoka K., Nakagawa T., Pharm. Res., 15, 1007-1011 (1998)

6) Ito T., Yamaoka K., Nakagawa T., J. Pharm. Pharmacol., 49, 1189-
1194 (1997).

7) Sawai Y., Yamaoka K., Ito T., Nakagawa T., Biol. Pharm. Bull., 20, 1313 -1316 (1997).

8) Ueda S., Yamaoka K., Nakagawa T., J. Pharm. Pharmacol., 51, 585589 (1999).

9) Weiss M., Forster W., Eur. J. Clin. Pharmacol., 16, 287-293 (1979).

10) Weiss M., J. Pharmacokinet. Biopharm., 11, 63-75 (1983).

11) Yamaoka K., Nakagawa T., Tanaka H., Chem. Pharm. Bull., 33, 784794 (1985).

12) Yano Y., Yamaoka K., Tanaka H., Chem. Pharm. Bull., 37, 10351038 (1989).

13) Yano Y., Yamaoka K., Yasui H., Nakagawa T., J. Pharmacokinet. Biopharm., 19, 71-84 (1991).

14) Yamaoka K., Kanba M., Toyoda Y., Yano Y., Nakagawa T., J. Pharmacokinet. Biopharm., 18, 545-559 (1990).

15) Hamada A., Fukushima S., Saneyoshi M., Shimizu S., Kawaguchi T., Nakano M., Biol. Pharm. Bull., 19, 729-732 (1996).

16) Christophidis N., Vajda F. J. E., Lucas I., Drummer O., Moon W. J., Louis W. J., J. Clin. Pharmacokinet., 3, 330-336 (1978).

17) Sullivan H. R., Billings R. E., McMahon R. E., J. Antibiot., 22, $195-$ 200 (1969).

18) Brian D., Tim M., Pharm. Res., 10, 1093-1095 (1993).

19) Colburn W. A., J. Pharmacokinet. Biopharm., 7, 407-415 (1979). 